\title{
A importância da enfermagem no pós-operatório de mulheres mastectomizadas com dissecção de linfonodos axilares: revisão integrativa
}

\author{
The importance of nursing in the post-operatory of mastectomized women with dissection of \\ axillary lymph nodes: integrative review \\ La importância de la enfermeria em el postoperatorio de mujeres mastectomizadas com disección \\ de nódulos linfáticos axilares: revisión integradora
}

Recebido: 17/05/2021 | Revisado: 23/05/2021 | Aceito: 26/05/2021 | Publicado: 10/06/2021

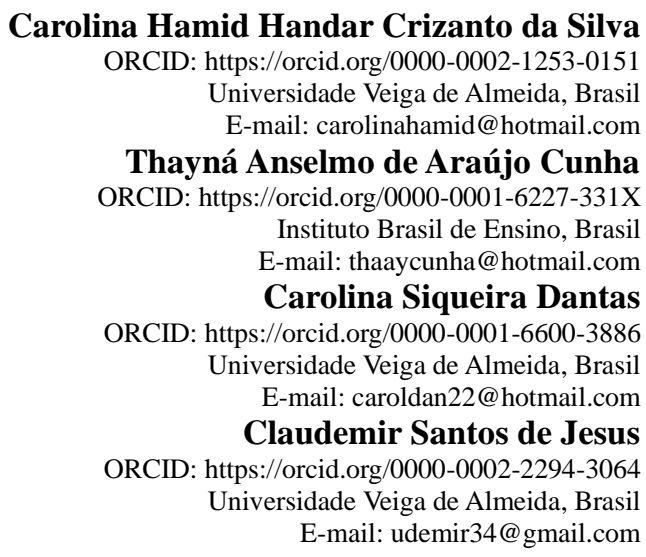

\begin{abstract}
Resumo
O estudo tem por objetivo reconhecer a importância do enfermeiro no período pós-operatório para auxiliar mulheres na reabilitação da mastectomia com dissecção dos linfonodos axilares. Trata-se de uma revisão integrativa, que se deu por um levantamento na base de dados da Biblioteca Virtual em Saúde, onde encontram-se disponíveis dados da LILACS, SciELO, Bdenf, entre outros. Diante da análise dos dados coletados, aplicando-se os critérios de inclusão e exclusão, emergiram-se as categorias: A enfermagem nas recomendações a mulheres com déficit de conhecimento submetidas a mastectomia e Acolhimento a mulheres com distúrbio de imagem corporal. Observou-se que os cuidados realizados pela equipe de enfermagem em mulheres no período de reabilitação pós-mastectomia, proporcionam grandes benefícios quando empregados em conjunto a ações educativas, orientação e escuta ativa. Além disso, a rede de apoio - amigos, familiares e profissionais de saúde - é de fundamental importância na aceitação da autoimagem e aderência a recuperação pós-operatória.
\end{abstract}

Palavras-chave: Cuidados de enfermagem; Reabilitação; Mastectomia.

\begin{abstract}
The study aims to recognize the importance of nurses in the postoperative period to assist women in the rehabilitation of mastectomy with dissection of axillary lymph nodes. This is an integrative review, which took place through a survey in the database of the Virtual Health Library, where data from LILACS, SciELO, Bdenf, among others, are available. In view of the analysis of the data collected, applying the inclusion and exclusion criteria, the following categories emerged: Nursing in the recommendations to women with knowledge deficits submitted to mastectomy and Reception to women with body image disorder. It was observed that the care provided by the nursing team to women in the post-mastectomy rehabilitation period, provides great benefits when used together with educational actions, guidance and active listening. In addition, the support network - friends, family and health professionals - is of fundamental importance in accepting self-image and adhering to postoperative recovery.
\end{abstract}

Keywords: Nursing care; Rehabilitation; Mastectomy.

\section{Resumen}

El estudio tiene como objetivo reconocer la importancia de las enfermeras en el postoperatorio para ayudar a las mujeres en la rehabilitación de la mastectomía con disección de los ganglios linfáticos axilares. Se trata de una revisión integradora, que se realizó a través de una encuesta en la base de datos de la Biblioteca Virtual en Salud, donde se encuentran disponibles datos de LILACS, SciELO, Bdenf, entre otros. A la vista del análisis de los datos recolectados, aplicando los criterios de inclusión y exclusión, surgieron las siguientes categorías: Enfermería en las 
recomendaciones a mujeres con déficit de conocimiento sometidas a mastectomía y Recepción a mujeres con trastorno de la imagen corporal. Se observó que la atención brindada por el equipo de enfermería a las mujeres en el período de rehabilitación posmastectomía, brinda grandes beneficios cuando se utiliza junto con acciones educativas, orientación y escucha activa. Además, la red de apoyo --amigos, familiares y profesionales de la salud-- es de fundamental importancia en la aceptación de la autoimagen y la adherencia a la recuperación postoperatoria.

Palabras clave: Cuidado de enfermera; Rehabilitación; Mastectomía.

\section{Introdução}

Mundialmente, o câncer de mama é aquele que mais acomete mulheres, excetuando-se o tumor de pele não melanoma. No ano de 2018, houve 2,1 milhões de casos novos, o que equivale a 11,6\% de todos os cânceres estimados. No Brasil, calcula-se que, a cada 100 mil mulheres, ocorram 61,61 casos novos (Instituto Nacional de Câncer José Alencar Gomes da Silva [INCA], 2019).

De acordo com o INCA (2019), o câncer de mama é resultado do crescimento desordenado de células oriundas de alterações genéticas decorrentes de fatores de risco hereditários, genéticos, ambientais e comportamentais. Diversos aspectos devem ser levados em consideração quando se determina o prognóstico de um paciente com câncer de mama, sendo os dois mais importantes o tamanho do tumor e a disseminação para linfonodos axilares.

O estado dos linfonodos é o fator prognóstico mais decisivo no câncer de mama. Em meados dos anos 1990, a biópsia de linfonodos sentinela (BLS) surgiu como alternativa menos invasiva do que a dissecação dos linfonodos axilares (DLA). O linfonodo sentinela, que é o primeiro linfonodo na cadeia linfática a receber drenagem do tumor primário da mama, é identificado, excisado e enviado para análise patológica. Quando este linfonodo é positivo, o cirurgião prossegue com a DLA imediata (Smeltzer \& Bare, 2014).

Atualmente, a BLS é tida como o padrão de cuidado para o tratamento de câncer de mama, posto que a DLA está associada à morbidade potencial, que se traduz em linfedema, mobilidade diminuída do braço e alterações sensoriais (Smeltzer $\&$ Bare, 2014).

Assim sendo, a cirurgia oncológica permanece como um dos métodos de tratamento mais indicados, podendo se dar por meio de uma abordagem invasiva ou não, a depender do estadiamento do câncer. A principal meta é o ganho no controle local da doença, seja por meio de uma mastectomia radical modificada, seja por mastectomia total, com realização de BLS.

Para Smeltzer e Bare (2014), a mastectomia radical modificada é um procedimento cirúrgico para a remoção de todo o tecido mamário, incluindo aréola e partes dos linfonodos axilares que também são removidos na DLA. Já a mastectomia total remove a mama e o complexo mamilo-aréola, mas não se realiza a DLA, uma vez que em cânceres de mama invasivos é feita a BLS.

$\mathrm{Na}$ recuperação pós-operatória de mulheres submetidas à mastectomia com DLS, a orientação pela equipe de enfermagem tem o propósito de educar e preparar a paciente para os tratamentos, reduzindo o medo e a ansiedade, promovendo a imagem corporal positiva dessa mulher, a fim de reduzir o impacto das manifestações sensoriais pós-operatórias (Alves et al., 2011).

Diante disso, o presente estudo tem por finalidade analisar a importância dos cuidados de enfermagem essenciais no auxílio do pós-operatório de mulheres submetidas à mastectomia com DLA.

\section{Metodologia}

Trata-se de um estudo de revisão integrativa, a qual é definida por Souza et al. (2010) como uma abordagem metodológica abrangente, que permite a inclusão de estudos experimentais ou não experimentais, para que se possa compreender de maneira completa o fenômeno analisado. Encerra uma ampla amostra e multiplicidade de propostas, gerando 
um panorama consistente sobre conceitos complexos, teorias ou problemas de saúde relevantes para a enfermagem. Desse modo, esta pesquisa examinou publicações relacionadas à temática com o fito de contribuir para a discussão a respeito dos cuidados de enfermagem no pós-operatório de mulheres submetidas à mastectomia com DLA.

Na primeira etapa, formulou-se a questão norteadora da pesquisa: Qual é a importância da assistência de enfermagem no processo de reabilitação da mulher mastectomizada? A segunda etapa correspondeu à busca de literatura na base de dados da Biblioteca Virtual em Saúde, de onde se tem acesso a publicações disponíveis na biblioteca on-line Scientific Eletronic Library Online (SciELO), Base de Dados de Enfermagem (BDENF), Literatura Latino-Americana e do Caribe em Ciências da Saúde (LILACS), Medical Literature Analysis and Retrieval System Online (MEDLINE), entre outras. Foram estabelecidos como critérios de inclusão: materiais de texto completo, em português, com recorte temporal de cinco anos (2016 a 2021); e como critérios de exclusão: os artigos editoriais. Empregaram-se as palavras-chave "cuidados de enfermagem", "reabilitação" e "mastectomia", almejando encontrar títulos, resumos e assuntos. Aplicou-se o operador booleano "AND" para se efetuar a combinação das palavras e equações, como ilustra o Quadro 1.

Quadro 1: Cruzamento dos descritores.

\begin{tabular}{|c|c|c|c|c|c|c|c|c|}
\hline DESCRITORES & TOTAL & $\begin{array}{l}\mathrm{F} \\
\mathrm{I} \\
\mathrm{L} \\
\mathrm{T} \\
\mathrm{R} \\
\mathrm{O}\end{array}$ & $\begin{array}{l}\mathrm{S} \\
\mathrm{E} \\
\mathrm{L} \\
\mathrm{E} \\
\mathrm{C} \\
\tilde{\mathrm{A}} \\
\mathrm{O}\end{array}$ & $\begin{array}{l}2 \\
0 \\
1 \\
6\end{array}$ & $\begin{array}{l}2 \\
0 \\
1 \\
7\end{array}$ & $\begin{array}{l}2 \\
0 \\
1 \\
8\end{array}$ & $\begin{array}{l}2 \\
0 \\
1 \\
9\end{array}$ & $\begin{array}{l}2 \\
0 \\
2 \\
0\end{array}$ \\
\hline $\begin{array}{l}\text { Mastectomia AND Cuidados de Enfermagem AND } \\
\text { Reabilitação }\end{array}$ & 100 & 2 & 0 & 0 & 0 & 0 & 0 & 0 \\
\hline Mastectomia AND Cuidados de Enfermagem & 587 & 14 & 6 & 1 & 1 & 0 & 2 & 2 \\
\hline Mastectomia AND Reabilitação & 5.920 & 31 & 5 & 0 & 1 & 0 & 2 & 2 \\
\hline TOTAL & 6.607 & 47 & 11 & 1 & 2 & 0 & 4 & 4 \\
\hline
\end{tabular}

Fonte: Autores.

Quando utilizados os descritores "mastectomia" AND "cuidados de enfermagem", foram identificados 587 estudos. Ao se aplicarem os filtros de disponibilidade na íntegra, idioma e recorte temporal, atinentes aos critérios de inclusão, foram encontrados 14 estudos. Seguindo-se à leitura de títulos e resumos, verificou-se que somente seis estudos contemplavam o assunto de interesse.

No segundo cruzamento, adotaram-se os descritores "mastectomia" AND "reabilitação", resultando em 5.920 publicações. Após a aplicação dos filtros, realizou-se a leitura prévia do título e resumo de 31 destas publicações, das quais apenas cinco se referiam à temática.

Por fim, procedeu-se a um terceiro cruzamento com os descritores "mastectomia" AND "cuidados de enfermagem" AND "reabilitação", localizando-se 100 publicações. Depois de aplicados os filtros, foi feita a leitura prévia do resumo de dois artigos. Constatou-se que apenas se relacionava com a temática, contudo tal publicação já havia sido selecionada em um cruzamento anterior. Desse modo, não se incluiu nenhuma outra publicação ao se aplicar o cruzamento das palavras-chave.

Ao todo, foram encontrados 6.607 estudos, dos quais somente 47 estavam de acordo com os critérios de inclusão, e destes, 36 não abordavam o tema. Foram eleitos, então, 11 estudos, sendo um de 2016, dois de 2017, quatro de 2019 e quatro de 2020 . 
As informações dos materiais selecionados são descritas na terceira fase.

$\mathrm{Na}$ quarta e última fase da presente pesquisa, que consistiu na análise e interpretação dos resultados obtidos, elaboraram-se duas categorias reflexivas e descritivas para análise dos dados, intituladas: “A enfermagem nas recomendações a mulheres com déficit de conhecimento submetidas à mastectomia" e "Acolhimento a mulheres com distúrbio de imagem corporal".

\section{Resultados}

Foi realizado um levantamento bibliográfico nas bases de dados da Biblioteca Virtual em Saúde, aplicando-se os critérios de inclusão descritos na metodologia do presente estudo, com o intuito de captar publicações sobre a referida temática, conforme demonstra o Quadro 2.

Quadro 2 - Levantamento estrutural dos artigos selecionados com a temática "a importância da enfermagem no pósoperatório de mastectomia com DLA”.

\begin{tabular}{|c|c|c|c|c|c|c|c|}
\hline Periódico & Base de dados & Ano & Local & Autores & Título & $\begin{array}{l}\text { Tipo de } \\
\text { Pesquisa }\end{array}$ & Tipo de Produção \\
\hline Rev enferm UERJ & LILACS & 2020 & RJ & $\begin{array}{l}\text { PANOBIANC } \\
\text { O, F. C. N. P. } \\
\text { et Al. }\end{array}$ & $\begin{array}{c}\text { Assistência de Enfermagem } \\
\text { em núcleo de reabilitação: o } \\
\text { papel do enfermeiro }\end{array}$ & Qualitativa & Artigo Descritivo \\
\hline $\begin{array}{l}\text { Ver enferm } \\
\text { Referãncia }\end{array}$ & BDENF & 2020 & Portugal & $\begin{array}{l}\text { MERÊNCIO, } \\
\text { Kátia. } \\
\text { VENTURA, } \\
\text { Maria. } \\
\end{array}$ & \begin{tabular}{|c|} 
Vivência da mulher \\
mastectomizada: a \\
enfermagem de reabilitação \\
na promoção da autonomia
\end{tabular} & Qualitativa & $\begin{array}{c}\text { Artigo } \\
\text { fenomenológico }\end{array}$ \\
\hline Ecc Anna Nery & $\begin{array}{l}\text { LILACS } \\
\text { BDENF }\end{array}$ & 2020 & RJ & $\begin{array}{c}\text { PAIVA, E. S. M. } \\
\text { M. A. et Al. }\end{array}$ & \begin{tabular}{|c|} 
Cuidados de enfermagem na \\
perspectiva do mundo da \\
vida da mulher- que- \\
vivência-linfedema- \\
decorrente-do-tratamento- \\
de-câncer-de-mama \\
\end{tabular} & Qualitativa & $\begin{array}{c}\text { Artigo } \\
\text { fenomenológico }\end{array}$ \\
\hline Revista Nursing & $\begin{array}{l}\text { LILACS } \\
\text { BDENF }\end{array}$ & 2020 & SP & $\begin{array}{c}\text { SCOFANO, B.S. } \\
\text { et. Al. }\end{array}$ & $\begin{array}{l}\text { Ações/plano de alta da } \\
\text { enfermagem à mulher } \\
\text { submetida à mastectomia }\end{array}$ & Qualitativa & Artigo Descritivo \\
\hline Saúde e Pesquisa & LILACS & 2019 & PR & $\begin{array}{l}\text { DINIZ, A. C. C. } \\
\text { N. et Al. }\end{array}$ & $\begin{array}{c}\text { Aspectos Comportamentais } \\
\text { da Mulher Mastectomizada } \\
\text { e a Ocorrência de } \\
\text { Complicações no Pós- } \\
\text { operatório }\end{array}$ & Quantitativa & Artigo Descritivo \\
\hline Saúde e Pesquisa & LILACS & 2019 & MG & $\begin{array}{c}\text { OLIVEIRA, T.R. } \\
\text { et. Al. }\end{array}$ & $\begin{array}{c}\text { Câncer de mama e imagem } \\
\text { corporal: impacto dos } \\
\text { tratamentos no olhar de } \\
\text { mulheres mastectomizadas }\end{array}$ & Qualitativo & Artigo Descritivo \\
\hline Ver Enferm UFPE & BDENF & 2019 & PE & $\begin{array}{l}\text { TRESCHER, } \\
\text { A.R. G. et Al. }\end{array}$ & $\begin{array}{c}\text { Necessidades das Mulheres } \\
\text { com Câncer de Mama no } \\
\text { pré-operatório }\end{array}$ & Qualitativa & Artigo Descritivo \\
\hline $\begin{array}{c}\text { Revista de Pesquisa: } \\
\text { Cuidado é Fundament } \\
\text { al Online }\end{array}$ & LILACS BDENF & 2019 & $\mathrm{SC}$ & URIO, A. et. Al. e & $\begin{array}{c}\text { O caminho do diagnóstico à } \\
\text { reabilitação: os sentimentos } \\
\text { e rede de apoio das mulheres } \\
\text { que vivenciam o câncer e a } \\
\text { mastectomia. }\end{array}$ & Qualitativa & Artigo Descritivo \\
\hline Cienc Cuid Saúde & LILACS BDENF & 2017 & RS & $\mid \begin{array}{c}\text { TIMM, P. B.P. et } \\
\text { Al. }\end{array}$ & $\begin{array}{c}\text { A Imagem Corporal na ótica } \\
\text { de Mulheres Após } \\
\text { Mastectomia }\end{array}$ & Qualitativo & Artigo Descritivo \\
\hline
\end{tabular}




\begin{tabular}{|c|c|c|c|c|c|c|c|}
\hline $\begin{array}{c}\text { Revista de Pesquisa } \\
\text { (Universidade Federal } \\
\text { do Estado do Rio de } \\
\text { Janeiro, Online) }\end{array}$ & LILACS BDENF & 2017 & RJ & $\begin{array}{c}\text { DIAS, L.V. et. } \\
\text { Al. }\end{array}$ & $\begin{array}{c}\text { Mulher mastectomizada por } \\
\text { câncer de mama: vivência } \\
\text { das atividades cotidianas }\end{array}$ & Qualitativo & Artigo Descritivo \\
\hline Cienc Cuid Saude & LILACS BDENF & 2016 & CE & $\begin{array}{c}\text { ALMEIDA, C. } \\
\text { R.M. et Al. }\end{array}$ & $\begin{array}{c}\text { Aspectos que Podem } \\
\text { vida da Mulher } \\
\text { Mastectomizada }\end{array}$ & Qualitativa & Artigo Descritivo \\
\hline
\end{tabular}

Fonte: Autores.

Quanto ao tipo de pesquisa, compuseram a amostra um estudo descritivo, quantitativo, de corte transversal (um); estudo descritivo, qualitativo (oito); estudo fenomenológico, qualitativo (dois).

Destaca-se que o delineamento da pesquisa se baseou no nível de evidência de estudos descritivos (não experimentais) ou com abordagem qualitativa.

\section{Discussão}

Dentre as condutas terapêuticas mais utilizadas para o tratamento do câncer de mama está o tratamento cirúrgico, representado, em grande parte, pela mastectomia com DLA. Frente aos resultados, é correto afirmar que o procedimento cirúrgico pode acarretar algumas limitações à paciente, tanto de cunho psicológico como de adaptação ao novo estilo de vida. Portanto, torna-se imprescindível que a equipe de enfermagem inicie seus cuidados com um atendimento humanizado, acolhedor e educativo, proporcionando oportunidades de melhoria da qualidade de vida. Com base nesses dados, emergiram as categorias que se seguem.

\section{A enfermagem nas recomendações a mulheres com déficit de conhecimento submetidas à mastectomia}

O período pós-operatório é marcado pela esperança de cura, mas também pelo medo, pois a mulher precisa enfrentar as permanentes alterações do seu corpo. Para Merêncio e Ventura (2020), a mulher mastectomizada atravessa um processo longo que suscita inúmeras implicações a nível físico, psicológico e social. É de fundamental importância que os enfermeiros direcionem intervenções para a promoção da independência e autonomia dessas mulheres, informando todos os cuidados necessários com o membro homolateral, potencializando na mulher a capacidade de recuperar a funcionalidade do braço e do ombro.

As autoras acrescentam que, no plano de cuidado estabelecido, a informação deve chegar à mulher mastectomizada e à sua família de um modo que estimule a discussão e que possam ser colocadas em pauta possíveis dúvidas e questões. Isto reitera a importância da atenção do enfermeiro à individualidade de cada mulher no processo de aprendizagem e reabilitação (Merêncio \& Ventura, 2020).

Urio et al. (2019) relatam que, no decorrer das entrevistas de sua pesquisa, a equipe de saúde não foi citada como rede de apoio, muito embora esta tenha papel essencial no apoio e na educação continuada da mulher. Sob esta ótica, cabe à equipe de enfermagem orientar e ouvir a mulher e seus familiares, esclarecendo todo o processo a ser vivenciado.

Panobianco et al. (2020) comentam que as ações de enfermagem podem ser percebidas em atividades grupais, em que são discutidas questões relativas ao enfrentamento do câncer, dúvidas, angústias e bons resultados. Esse relacionamento entre paciente e enfermeiro permite ao profissional ampliar sua assistência e colaborar com mais assertividade no enfrentamento da doença, em conjunto com os familiares. Ademais, é nesses encontros que se estimula o autocuidado, valoriza-se o indivíduo e se promove o crescimento individual dos pacientes. $\mathrm{O}$ acolhimento por parte do enfermeiro é apontado como fator crucial para 
a aderência de pacientes aos grupos de reabilitação, confirmando a importância do profissional no processo de reabilitação pósoperatória.

Em geral, as informações e ações educativas, realizadas tanto pela equipe de enfermagem quanto pelos demais componentes da equipe multiprofissional, são vivenciadas nas consultas e atividades grupais. A promoção da qualidade de vida das mulheres que retiram a mama pode advir do apoio e do processo educacional, fomentados como forma de enfrentamento do processo de adoecimento, tratamento e autocuidado (Trescher et al., 2019).

Reforçou-se, através desta pesquisa, que o acolhimento e a escuta ativa, no período de reabilitação dessas mulheres, possibilitam uma comunicação efetiva entre o profissional, a mulher e a família. Os autores supracitados complementam dizendo que esse processo contribui para o reconhecimento do momento vivenciado pela mulher e seus familiares, e para a desconstrução da rotina mecânica de cuidados, propiciando uma atuação mais humanizada.

Com o estudo de Scofano et al. (2020), é possível salientar que a eficiência e eficácia das ações de enfermagem sofrem ação direta do vínculo e compreensão estabelecidos entre profissional, paciente e familiar. No estudo em questão, identificaram-se 39 intervenções de enfermagem e 42 resultados voltados à mastectomia, cuja base foi a abordagem integral, humanizada e individualizada da mulher. Tais intervenções atentam para as reais necessidades da mulher a partir das problemáticas apresentadas por elas, principalmente em questões emocionais (medo, anseios, tristeza). Isto só realça a relevância e a amplitude das ações de enfermagem nesse contexto de saúde.

Trescher et al. (2019) e Merêncio e Ventura (2020) fazem também uma reflexão sobre a importância do apoio familiar no enfrentamento da doença e tratamento. A presença de uma pessoa de confiança confere ao paciente conforto e proteção, auxiliando no processo de aceitação da doença. O familiar deve ser envolvido em todas as etapas do tratamento para que, mesmo em casa, longe da unidade de atendimento, a reabilitação seja incentivada.

Segundo Diniz et al. (2019), o profissional de saúde deverá desenvolver atividades educativas com o objetivo de fornecer informações relacionadas aos cuidados necessários após a mastectomia, que ajudem a mulher no processo de recuperação. O enfermeiro tem o dever de acompanhar essas atividades para que as melhorias do processo de reabilitação, com base nos erros e na falta de conhecimento, possam ser alcançadas. No entanto, é primordial que as mulheres compreendam a importância de se cumprirem as medidas diárias para a minimização da probabilidade de complicações pós-operatórias.

Dentre os cuidados recomendados às mulheres mastectomizadas, estão: evitar a exposição ao sol; não apertar o lado do membro operado; ter cuidado para não sofrer queimaduras, arranhões e cortes; não receber injeções, vacinas ou retirar sangue no membro superior homolateral à cirurgia; evitar retirar cutícula; não carregar peso; exercitar os membros superiores; entre outros (Diniz et al., 2019).

\section{Acolhimento a mulheres com distúrbio de imagem corporal}

Após a mastectomia, a mulher enfrenta algumas dificuldades, sobretudo com relação à alteração da sua imagem corporal. Timm et al. (2017) retratam bem a realidade da mulher ao ver o seu novo corpo, ressaltando o sofrimento e as mudanças relevantes. A mastectomia, nesse contexto, é definida como mutilação, e a resposta a esse processo é individual, consoante aos fatores de vida daquela mulher como idade, percepção da autoimagem e estado emocional. Com isso, além dos cuidados próprios à cirurgia, as mulheres mastectomizadas precisam de apoio emocional que promova melhor compreensão e aceitação de sua nova autoimagem, tornando-se evidente o valor do trabalho da equipe de enfermagem, multiprofissional e familiar no processo de reabilitação.

Urio et al. (2019) enfatizam também a importância do apoio na vivência dessa experiência, quando alegam que a motivação para o enfrentamento da doença e seu tratamento tem correlação com o apoio recebido. A partir do diagnóstico, 
inúmeros sentimentos são despertados na mulher. Por vezes, o sofrimento psíquico que o diagnóstico do câncer de mama gera mais consequências do que o tratamento e a necessidade de retirada da mama.

Oliveira et al. (2019) elucidam que a desfiguração da mama, como consequência da doença, altera a percepção corporal da mulher, o que torna este tipo de neoplasia uma das mais temidas pelas mulheres, uma vez que o modo como o corpo é percebido e representado influencia enormemente a construção da autoimagem. Mudanças deste tipo trazem diversos questionamentos acerca da identidade feminina, pois a mama é um órgão associado à fertilidade, feminilidade e sexualidade. A aceitação é uma parte dolorosa do processo, e, nesse sentido, a rede de apoio faz total diferença na forma como a mulher irá passar por todo o tratamento e enfrentamento da doença.

Dentro desse processo, a primeira grande dificuldade da mulher é se reconhecer em frente ao espelho, buscando a aceitação de seu novo corpo, em que se mostra ausente a parte que, culturalmente, simboliza a feminilidade. Segundo o estudo, esse primeiro contato tem impacto negativo, provoca tristeza e reflete uma imagem corporal negativa (Timm et al., 2017).

Nos casos em que a imagem corporal se distancia do que é considerado normal pela sociedade, a mulher se enxerga de maneira inferiorizada. Essa insatisfação está relacionada a fatores culturais e socioeconômicos, e converte em isolamento social e falta de autocuidado. Acredita-se que há uma associação entre a insatisfação com a imagem corporal e o surgimento de sintomas depressivos, dor e sofrimento psíquico (Paiva et al., 2020).

Tal fato revela as limitações que a mulher mastectomizada enfrentará perante a sociedade. O preconceito social constrange essa mulher, e isso dificulta ainda mais o enfrentamento da nova realidade, contribuindo para o preconceito da própria mulher para com o seu corpo. Por esta razão, o profissional de saúde deve auxiliá-la no enfrentamento desses desafios, buscando incentivá-la a retomar as atividades sociais e funcionais, elevando sua autoestima e autoconfiança (Paiva et al., 2020).

Para Dias et al. (2017), as mulheres mastectomizadas descobrem variadas formas de lidar com as dificuldades de seu cotidiano; e destacam a rede de apoio. Em vista de todo o enfrentamento da doença, algumas mulheres passam a apresentar sintomas psicológicos como a depressão, repercutindo-se na capacidade funcional das mulheres que, muitas vezes, deixam de praticar atividade física após o diagnóstico da neoplasia.

O apoio social proporciona métodos de enfrentamento emocional e tem como finalidade a proteção da saúde humana, podendo ser trabalhado através de uma rede de apoio que engloba família, amigos e equipe multiprofissional. Para Almeida et al. (2016), além do apoio familiar e social, surge como subcategoria o apoio do profissional, que é vivenciado pela mulher nas consultas e atividades com enfermeiros, os quais conseguem auxiliar no enfrentamento emocional, promovendo proteção e escuta ativa. Assim, em redes de apoio, estes profissionais se tornam indispensáveis para o fortalecimento pessoal e motivacional das mulheres.

O estudo de Timm et al. (2017) expõe o depoimento de mulheres sobre o apoio recebido de familiares e amigos, assim como a sensação de conforto e confiança em relação ao profissional de enfermagem. Esta rede de apoio contribui para o fortalecimento dessa mulher, para que mantenha uma vida social ativa, e minimiza as tensões emocionais. Algumas mulheres relatam a importância da fé para o enfrentamento dos problemas, tendo em vista que pode ser uma grande aliada nos momentos difíceis.

Para Almeida et al. (2016), em redes de apoio, o profissional deve ser capaz de atender às demandas destas pacientes sob a perspectiva do cuidado humanizado e acolhedor, satisfazendo as necessidades do indivíduo de maneira integral. Este processo estimula o fortalecimento pessoal. Para tal, é preciso o engajamento da equipe de enfermagem frente aos pacientes oncológicos, respeitando cada um como ser único, incorporando os cuidados como forma de fortalecer as redes de apoio da mulher mastectomizada. 
Portanto, salienta-se a necessidade de o enfermeiro comprometer-se com a manutenção do cuidado humanizado, contribuindo para a melhoria das condições de vida, adaptação à nova realidade, prevenção de danos e incentivo da autonomia dessa mulher diante das diversidades inerentes ao adoecimento (Paiva et al., 2020).

\section{Considerações Finais}

O câncer de mama é um dos tipos de cânceres com maior taxa de mortalidade, entretanto há cura para a patologia por meio de procedimentos cirúrgicos e tratamentos adjuvantes. Em caso de cirurgia radical (mastectomia com DLA), a mulher vivencia um misto de incerteza e insegurança ante o desconhecido, o que afeta diretamente o seu estado emocional, físico e psicológico. Por conseguinte, o enfermeiro tem um papel fundamental no pós-operatório da mulher submetida à mastectomia, especialmente no sentido educativo, com o propósito de ofertar à mulher melhores condições na retomada das atividades rotineiras, promovendo autonomia e segurança emocional.

Nas consultas de enfermagem e atividades grupais, o enfermeiro pode facilitar o gerenciamento dos cuidados pósoperatórios com o membro homolateral, bem como contribuir com estratégias de educação em saúde - prestando um atendimento humanizado e acolhedor à paciente, para que esta consiga superar os desafios do processo da reabilitação. Cabe à enfermagem a orientação quanto aos anseios da paciente e a promoção de uma melhora na qualidade de vida através das recomendações.

Destarte, ressalta-se a importância do enfermeiro no que diz respeito à compreensão da percepção que a mulher tem de si mesma, em relação à sua imagem corporal. Esse processo se inicia quando o profissional consegue identificar estratégias que aumentem a autoestima e melhorem a qualidade de vida da paciente, de modo que ela possa encarar e enfrentar as dificuldades.

O enfermeiro, como líder de todo esse processo, deve considerar as individualidades e singularidades de cada mulher e sua família, para melhor auxiliá-las no enfrentamento das dificuldades. Deve, também, integrá-las no processo de recuperação, para conhecerem os cuidados com seu corpo, visto que pairam sobre elas e suas famílias preocupações sobre os cuidados no pós-operatório.

Desta forma, os resultados obtidos por este estudo possibilitam o entendimento da relevância da equipe de enfermagem, atuando com uma abordagem humanizada, a fim de acolher os pacientes, envolvendo-os no tratamento e ofertando suporte informativo durante as consultas de enfermagem e grupos educativos.

\section{Referências}

Almeida, N. G. de, Cruz, A. M., Rodrigues, D. P, Moreira, T. M. M., Figueiredo, J. V., Fialho, A. V. de M. (2016). Aspectos que podem influenciar a qualidade de vida da mulher mastectomizada. Ciênc. cuid. saúde, 15(3), 452-459. https://doi.org/10.4025/cienccuidsaude.v15i3.31526

Alves, P. C., Barbosa, I. C. F. J., Caetano, J. A., \& Fernandes, A. F. C. (2011). Cuidados de enfermagem no pré-operatório e reabilitação de mastectomia: Revisão narrativa da literatura. Revista Brasileira de Enfermagem, 64(4), 732-737. https://doi.org/10.1590/S0034-71672011000400016

Dias, L. V., Muniz, R. M., Viegas, A. C., Cardoso, D. H., Amaral, D. E. D., \& Pinto, B. K. (2017). Mulher mastectomizada por câncer de mama: Vivência das atividades cotidianas. Rev. Pesqui. (Univ. Fed. Estado Rio J., Online), 9(4), 1074-1080. https://doi.org/10.9789/2175-5361.2017.v9i4.1074-1080

Diniz, F. S., Almeida, A. S., Campos, M. P. A., Carvalho, T. A., \& Nascimento, Q. S. (2019). Aspectos comportamentais da mulher mastectomizada e a ocorrência de complicações no pós-operatório. Saúde e pesquisa (Online), 2(2), 275-282. https://doi.org/10.17765/2176-9206.2019v12n2p275-282

Instituto Nacional de Câncer José Alencar Gomes da Silva (INCA). (2019). Estimativa 2020: Incidência de câncer no Brasil. https://www.inca.gov.br/publicacoes/livros/estimativa-2020-incidencia-de-cancer-no-brasil

Merêncio, K. M., \& Ventura, M. C. A. A. (2020). Vivência da mulher mastectomizada: A enfermagem de reabilitação na promoção da autonomia. Referência, 5(2), e19082. https://doi.org/10.12707/RIV19082

Oliveira, T. R., Corrêa, C. S. L., Weiss, V. F., Baquião, A. P. S. S., Carvalho, L. L., Grincenkov, F. R. S., \& Carvalho, S. M. (2019). Câncer de mama e imagem corporal: Impacto dos tratamentos no olhar de mulheres mastectomizadas. Saúde e pesquisa (Online), 12(3), 451-462. https://doi.org/10.17765/21769206.2019v12n3p451-462 
Research, Society and Development, v. 10, n. 6, e57210616177, 2021

(CC BY 4.0) | ISSN 2525-3409 | DOI: http://dx.doi.org/10.33448/rsd-v10i6.16177

Paiva, A. C. P. C., Elias, E. A., Souza, I. E. O., Moreira, M. C., Melo, M. C. S. C., \& Amorim, T. V. (2020). Cuidado de enfermagem na perspectiva do mundo da vida da mulher-que-vivencia-linfedema-decorrente-do-tratamento-de-câncer-de-mama. Esc. Anna Nery Rev. Enferm., 24(2), e20190176. https://doi.org/10.1590/2177-9465-EAN-2019-0176

Panobianco, M. S., Felipe, I. O., Canete, A. C. S., Nunes, L. C., \& Prado, M. A. S. (2020). Assistência de enfermagem em núcleo de reabilitação: O papel do enfermeiro. Rev. Enf. UERJ, 28, e51082. https://doi.org/10.12957/reuerj.2020.51082

Scofano, B. S., Lima, A. A., Silva, R. R., Penna, L. H. G., Andrade, K. B. S., Pinheiro, A. P. B. (2020). Ações/plano de alta da enfermagem à mulher submetida à mastectomia. Revista Nursing, 23(263), 3736-3744. http://www.revistanursing.com.br/revistas/263/pg79.pdf

Smeltzer, S. C., \& Bare, B. G . (2014). Brunner \& Suddart: Tratado de enfermagem médico-cirúrgica (12a ed.). Guanabara Koogan.

Souza, M. T.; Silva, M. D., \& Carvalho, R. (2010). Revisão integrativa: O que é e como fazer. Einstein, 8(1), 102-106. https://doi.org/10.1590/S167945082010RW1134

Timm, M. S., Perlini, N. M. O. G., Beuter, M., Prates, L. A., Birk, N. M., \& Piccin, C. (2017). A imagem corporal na ótica de mulheres após a mastectomia. Ciênc. cuid. saúde, 16 (1). https://doi.org/10.4025/cienccuidsaude.v16i1.30151

Trescher, G. P., Amante, L. N. A., Rosa, L. M., Girondi, J. B. R., Varela, A. I. S., Oro, J., Rolim, J. M., \& Santos, M. J. (2019). Necessidades das mulheres com câncer de mama no período pré-operatório. Rev. enferm. UFPE on line, 13(5), 1288-1294. https://periodicos.ufpe.br/revistas/revistaenfermagem/article/view/239229

Urio, A., Souza, J. B., Manorov, M., \& Soares, R. B. S. (2019). O caminho do diagnóstico à reabilitação: Os sentimentos e rede de apoio das mulheres que vivenciam o câncer e a mastectomia. Rev. Pesqui. (Univ. Fed. Estado Rio J., Online), 11(4), 1031-1037. https://doi.org/10.9789/2175-5361.2019.v11i4.10311037 